\title{
Estudio numérico y comparativo del efecto de turbulencia en codos y dobleces para distribución de agua sanitaria
}

\author{
Luis Fernando Toapanta-Ramos ${ }^{1}$ \\ Jorge Alejandro Zapata-Cautillo² \\ Andrea Isabel Cholango-Gavilanes ${ }^{3}$ \\ William Quitiaquez ${ }^{4}$ \\ César Nieto-Londoño ${ }^{5}$ \\ Zulamita Zapata-Benabithe ${ }^{6}$
}

Fecha de recepción: 21 de junio de 2019

Fecha de aprobación: 30 de septiembre de 2019

\section{Resumen}

En este artículo se presenta el estudio numérico y comparativo del efecto de turbulencia en codos y dobleces para diámetros de una pulgada, mediante CFD y bajo las mismas condiciones de trabajo (velocidad, presión y temperatura), para determinar la fluctuación en la turbulencia de energía cinética entre estos dos accesorios variando los modelos de turbulencia. Se emplearon dos metodologías para esta investigación, kappa-épsilon $(k-\varepsilon)$ y kappa-omega $(k-\omega)$. El método $(k-\varepsilon)$ se divide en tres modelos: estándar, RNG y realizable, en los cuales se genera turbulencia de energía cinética y de disipación. El método $(k-\omega)$ también posee tres variantes: estándar, SST, BSL. El trabajo presenta una mayor turbulencia para el método de $(k-\varepsilon)$ en energía cinética y de disipación bajo el modelo estándar tanto para codo como doblez, mientras que en el método $(k-\omega)$ se produce una

\footnotetext{
${ }^{1}$ M.Sc. Universidad Politécnica Salesiana (Quito, Ecuador). Itoapanta@ups.edu.ec. ORCID: 0000-0002-08384702 .

2 Universidad Politécnica Salesiana (Quito, Ecuador). acholangog@est.ups.edu.ec. ORCID: 0000-0001-93550957.

${ }^{3}$ Universidad Politécnica Salesiana (Quito, Ecuador). izapatac3@est.ups.edu.ec. ORCID: 0000-0001-50142021.

${ }^{4}$ M.Sc. Universidad Politécnica Salesiana (Quito, Ecuador). wquitiaquez@ups.edu.ec. ORCID: 0000-00019430-2082.

5 Ph.D. Universidad Pontificia Bolivariana (Medellín-Antioquia, Colombia). cesar.nieto@upb.edu.co. ORCID: 0000-0001-6516-9630.

6 Ph.D. Universidad Pontificia Bolivariana (Medellín-Antioquia, Colombia). zulamita.zapata@upb.edu.co. ORCID: 0000-0002-4497-4865.
} 
mayor turbulencia de energía cinética en el modelo BSL para ambos accesorios, al igual que en el método $(k-\varepsilon)$, el modelo estándar de $(k-\omega)$ representa una mayor turbulencia de frecuencia.

Palabras clave: codo; doblez; kappa-épsilon; kappa-omega; turbulencia.

\section{Numerical and Comparative Study of the Turbulence Effect on Elbows and Bends for Sanitary Water Distribution}

\section{Abstract}

This article presents the numerical and comparative study of the effect of turbulence on elbows and bends for the parameters of an inch, by means of CFD and under the same working conditions (velocity, pressure and temperature), to determine the fluctuation in turbulence of kinetic energy between these two accessories varying the turbulence models. Two methodologies were used for this investigation, kappa-epsilon $(k-\varepsilon)$ and kappa-omega $(k-\omega)$. The method $(k-\varepsilon)$ is divided into three models: standard, RNG and realizable, in which turbulence of kinetic energy and of dissipation is transformed. The method $(k-\omega)$ also has three variants: standard, SST, BSL. The work presents a greater turbulence for the method of $(k-\varepsilon)$ in kinetic energy and dissipation under the standard model for both elbow and bend, while in the method $(k-\omega)$ there is a greater turbulence of kinetic energy in the BSL model for both accessories, as in the method $(k-\varepsilon)$ the standard model of $(k-\omega)$ represents a greater frequency turbulence.

Keywords: bend; elbow; kappa-epsilon; kappa-omega; turbulence.

\section{Estudo numérico e comparativo do efeito de turbulência em joelhos e dobras para a distribuição de água sanitária}

\section{Resumo}

Neste artigo apresenta-se o estudo numérico e comparativo do efeito de turbulência em joelhos e dobras para diâmetros de uma polegada, mediante CFD e sob as mesmas condições de trabalho (velocidade, pressão e temperatura), para determinar a flutuação na turbulência de energia cinética entre estes dois acessórios variando os modelos de turbulência. Foram empregadas duas 
metodologias para esta pesquisa, kappa-épsilon $(k-\varepsilon)$ e kappa-ômega $(k-\omega)$. $O$ método $(k-\varepsilon)$ divide-se em três modelos: estândar, $R N G$ e realizável, nos quais se gera turbulência de energia cinética e de dissipação. $O$ método $(k-\omega)$ também possui três variantes: estândar, SST, BSL. O trabalho apresenta uma maior turbulência para o método de $(k-\varepsilon)$ em energia cinética e de dissipação sob 0 modelo estândar tanto para joelho como dobra, enquanto que no método $(k-\omega)$ se produz uma maior turbulência de energia cinética no modelo BSL para ambos os acessórios, ao igual que no método $(k-\varepsilon)$, o modelo estândar de $(k-\omega)$ representa uma maior turbulência de frequência.

Palavras chave: joelho; dobra; kappa-épsilon; kappa-ômega; turbulência.

\section{Para citar este artículo:}

L. F. Toapanta-Ramos, J. A. Zapata-Cautillo, A. I. Cholango-Gavilanes, W. Quitiaquez, C. Nieto-Londoño, and Z. Zapata-Benabithe, "Estudio numérico y comparativo del efecto de turbulencia en codos y dobleces para distribución de agua sanitaria," Revista Facultad de Ingeniería, vol. 28 (53), pp. 101-118, 2019. https://doi.org/10.19053/01211129.v28.n53.2019.10156.

Esta obra está bajo licencia internacional Creative Commons Reconocimiento 4.0

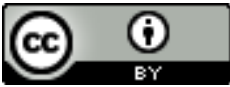


Estudio numérico y comparativo del efecto de turbulencia en codos y dobleces para distribución de agua sanitaria

\section{INTRODUCCIÓN}

El movimiento de los fluidos a través de conductos cerrados, los cambios de sección y, sobre todo, el cambio de dirección del flujo, son las causas que generan turbulencia. Mediante esta investigación se busca comprender el efecto de turbulencia en codos y doblez, para observar si la curvatura de estos objetos modifica la turbulencia. Se han realizado varios estudios sobre estas curvaturas, la mayoría de los cuales se centraron en la pérdida de presión entre la entrada y la salida del codo y el perfil de velocidad promediada en el tiempo, desde un punto de vista de la aplicación industrial.

Los fluidos turbulentos son fenómenos complejos estudiados por la mecánica de fluidos, la mayoría de los flujos existentes en la vida diaria son turbulentos. Este tipo de flujo es debido a la variación de presión y velocidad, por este motivo se conoció que la inestabilidad y el desorden resultantes no son las únicas propiedades definitorias de la turbulencia [1].

Según Davidson [2], los fluidos turbulentos aumentan la difusividad del agua, con lo cual se incrementa la resistencia del fluido (fricción en las paredes de la tubería) y la transferencia de calor tanto en flujos internos, como en canales y tuberías. El flujo turbulento es siempre tridimensional e inestable. Sin embargo, cuando las ecuaciones se promedian en el tiempo, se trata al flujo como bidimensional. Las ecuaciones tridimensionales de Reynolds promediadas de Navier-Stokes (RANS), se aplican para conocer el método de turbulencia [3].

Ikarashi et al. [4] llevaron a cabo varios estudios numéricos sobre los flujos en codos con alto número de Reynolds, para comprender su fenómeno. Los estudios anteriores se hicieron en codos de diferente radio, que van desde $\mathrm{R} / \mathrm{d}=1.0$ a $2.8, \mathrm{y}$ un número de Reynolds ( $R e=(4.3$ a 32$\left.) \cdot 10^{\wedge} 4\right)$, dando como resultado que las características de un flujo son independientes del número de Reynolds [5].

Zamani et al. [6] y su enfoque en los codos y la erosión, fijaron a estos como las partes más vulnerables de las tuberías de transporte, que corren el riesgo de fallar debido a la presencia de partículas de arena en el flujo turbulento. Se conoce que la existencia de partículas sólidas en tuberías de gas o líquido, como las partículas de arena, es una causa importante de erosión. Los codos son particularmente más 
propensos a la erosión, porque el impacto de las partículas sólidas remueve partes del material. Además, el cambio repentino en la dirección del flujo en los codos conduce a cambios considerables en la distribución de partículas en el flujo y, por consiguiente, a una mayor tasa de erosión. Por ejemplo, Lin et al. [7] mostraron que la tasa de erosión de los codos de $90^{\circ}$ es 50 veces mayor que en los tubos rectos.

En este artículo se hará un estudio numérico comparativo entre dos accesorios utilizados en los sistemas de tuberías, un codo estándar y doblez, utilizando los modelos de turbulencia kappa-épsilon y kappa-omega, para determinar la variación de energía cinética de turbulencia.

\section{MATERIALES Y MÉTODOS}

Los métodos utilizados para determinar turbulencia en codos y dobleces comienzan con el cálculo del número de Reynolds, con el cual se determina si el fluido se encuentra en estado laminar, turbulento o transitorio. Las ecuaciones de Navier Stokes (RANS) representan la velocidad, la presión, la temperatura y el transporte en el flujo, mas no las fluctuaciones turbulentas. Las tres ecuaciones de $(k-\omega)$ son las más utilizadas para un flujo turbulento y representan las propiedades

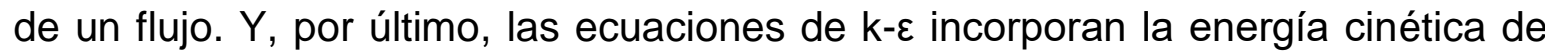
turbulencia $(k)$, la tasa de disipación de la turbulencia $(\varepsilon)$ y el coeficiente de viscosidad turbulenta $(\mu \mathrm{t})$ para un modelado matemático más acertado.

\section{A. El número de Reynolds}

Existen varios motivos para determinar si un fluido es turbulento o no. Según los estudios realizados por Zacarías et al. [8], el número de Reynolds crece al aumentar la velocidad, al igual que al utilizar tubería de diferentes diámetros o fluidos con viscosidades 0 densidades diferentes. Mediante procesos experimentales, se comprobó un flujo laminar para un número de Reynolds menor a 2000, flujo transitorio si se encuentra entre 2000 y 4000 , y flujo turbulento si supera los 4000 [8]. El número de Reynolds se representa con la fórmula (1).

$$
\operatorname{Re}=\frac{\rho \cdot v \cdot D}{\mu}
$$


Estudio numérico y comparativo del efecto de turbulencia en codos y dobleces para distribución de agua sanitaria

Donde: $\rho$, es la densidad; $\mathrm{v}$, la velocidad promedio del fluido; $\mathrm{D}$, diámetro interno del tubo; y $\mu$, viscosidad absoluta del fluido.

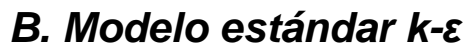

En la investigación realizada por Rumsey [9], se expresa que el modelado de turbulencia para las ecuaciones de Naiver-Stokes incluye muchas suposiciones y cierres simplificados, donde se han desarrollado varios modelos con los años, siendo todo deficiente de un modo $u$ otro. Por esta razón es que se han implementado varias metodologías y modelos de turbulencia en la dinámica de fluidos computacional.

Según la investigación realizada por Bayona et al. [10], el modelo k- $\varepsilon$ permite obtener escalas de longitud y velocidad de turbulencia. Las ecuaciones de transporte describen la energía cinética y la tasa de disipación de turbulencia.

Una de las ventajas del modelo $\mathrm{k}-\varepsilon$ es efectuar cálculos convergentes con resultados coherentes para distintos regímenes de flujo. En contraste con sus desventajas, el modelo no resulta preciso para flujos en rotación como fluidos en tubos circulares.

Las ecuaciones de transporte $k-\varepsilon$ estándar son:

$$
\begin{gathered}
\frac{\partial}{\partial t}(\rho \kappa)+\frac{\partial}{\partial x_{i}}\left(\rho \kappa \mu_{i}\right)=\frac{\partial}{\partial x_{j}}\left[\left(\mu+\frac{\mu_{t}}{\sigma_{\kappa}}\right) \frac{\partial \kappa}{\partial x_{j}}\right]+G_{\kappa}+G_{b}-\rho \varepsilon-Y_{M}+S_{\kappa} \\
\frac{\partial}{\partial t}(\rho \varepsilon)+\frac{\partial}{\partial x_{i}}\left(\rho \varepsilon \mu_{i}\right)=\frac{\partial}{\partial x_{j}}\left[\left(\mu+\frac{\mu_{t}}{\sigma_{\varepsilon}}\right) \frac{\partial \varepsilon}{\partial x_{j}}\right]+C_{1 \varepsilon} \frac{\varepsilon}{\kappa}\left(G_{\kappa}+C_{3 \varepsilon} G_{b}\right)-C_{2 \varepsilon} \rho \frac{\varepsilon^{2}}{\kappa}+S_{\varepsilon}
\end{gathered}
$$

En estas ecuación, $G_{\kappa}$ representa la generación de energía cinética de turbulencia debido a los gradientes de velocidad promedio; $G_{b}$ es la generación de energía cinética debido a la flotabilidad; $Y_{M}$ representa la contribución de la dilatación fluctuante en la turbulencia compresible a la tasa de disipación global; $C_{1 \varepsilon}, C_{2 \varepsilon}, C_{3 \varepsilon}$ son constantes; $\sigma_{\kappa}$ y $\sigma_{\varepsilon}$ son los números de Prandtl para $\kappa$ y $\varepsilon$, respectivamente; $S_{\kappa}$ y $S_{\varepsilon}$ son términos definidos por el usuario [11]. 


\section{Modelo RNG $k-\varepsilon$}

En este modelo, los elementos de movimiento de baja escala se eliminan sistemáticamente mediante la aplicación de herramientas estadísticas. Las ecuaciones de control del flujo de fluido se modifican con respecto a los elementos de gran movimiento. Este modelo de turbulencia considera el régimen superior con efectos bajos o nulos de la región amortiguadora.

El modelo RNG k- $\varepsilon$ no puede predecir el efecto de las esquinas, debido a la restricción en el uso de la rejilla gruesa cerca de la pared y las esquinas. Este modelo predice una buena concordancia con el medio poroso grande. Con el modelo RNG se produjeron mejores resultados que con el modelo k- $\varepsilon$ estándar, debido a que considera condiciones anisotrópicas complejas [12].

Las ecuaciones de transporte para el modelo k- $\varepsilon$ RNG son:

$$
\begin{gathered}
\frac{\partial}{\partial t}(\rho \kappa)+\frac{\partial}{\partial x_{i}}\left(\rho \kappa \mu_{i}\right)=\frac{\partial}{\partial x_{j}}\left[\left(\alpha_{\kappa} \mu_{e f f}\right) \frac{\partial \kappa}{\partial x_{j}}\right]+G_{\kappa}+G_{b}-\rho \varepsilon-Y_{M}+S_{\kappa} \\
\frac{\partial}{\partial t}(\rho \varepsilon)+\frac{\partial}{\partial x_{i}}\left(\rho \varepsilon \mu_{i}\right)=\frac{\partial}{\partial x_{j}}\left[\left(\alpha_{\varepsilon} \mu_{e f f}\right) \frac{\partial \varepsilon}{\partial x_{j}}\right]+C_{1 \varepsilon} \frac{\varepsilon}{\kappa}\left(G_{\kappa}+C_{3 \varepsilon} G_{b}\right)-C_{2 \varepsilon} \rho \frac{\varepsilon^{2}}{\kappa}-R_{\varepsilon}+S_{\varepsilon}
\end{gathered}
$$

Donde: $\alpha_{\varepsilon}$ y $\alpha_{\kappa}$, son números inversos de Prandtl para $\kappa$ y $\varepsilon$, respectivamente.

\section{Modelo realizable $k-\varepsilon$}

Los modelos realizables y RNG presentan características superiores que las del modelo estándar en cuanto a curvatura y rotación en flujos con corrientes turbulentas. Además, los estudios previos del modelo realizable han demostrado que este es ideal para validación de flujos separados y con características complejas.

El modelo realizable propuesto por Shih et al. [13] tenía la intención de abordar deficiencias de los modelos tradicionales mediante la adopción de una nueva fórmula de viscosidad Eddy, lo que implica una variable propuesta por Reynolds.

$$
\begin{gathered}
\frac{\partial}{\partial t}(\rho \kappa)+\frac{\partial}{\partial x_{j}}\left(\rho \kappa \mu_{j}\right)=\frac{\partial}{\partial x_{j}}\left[\left(\mu+\frac{\mu_{t}}{\sigma_{\kappa}}\right) \frac{\partial \kappa}{\partial x_{j}}\right]+G_{\kappa}+G_{b}-\rho \varepsilon-Y_{M}+S_{\kappa} \\
\frac{\partial}{\partial t}(\rho \varepsilon)+\frac{\partial}{\partial x_{j}}\left(\rho \varepsilon \mu_{j}\right)=\frac{\partial}{\partial x_{j}}\left[\left(\mu+\frac{\mu_{t}}{\sigma_{\varepsilon}}\right) \frac{\partial \varepsilon}{\partial x_{j}}\right]+\rho C_{1} S \varepsilon-\rho C_{2} \frac{\varepsilon^{2}}{\kappa+\sqrt{v \varepsilon}}+C_{1 \varepsilon} \frac{\varepsilon}{\kappa} C_{3 \varepsilon} G_{b}+S_{\varepsilon}
\end{gathered}
$$


Donde:

$$
\mathrm{S}=\sqrt{2 S_{i j} S_{i j}}
$$

\section{E. Modelo estándar (Wilcox) $k-\omega$}

Este modelo fue desarrollado por Wilcox, donde la cantidad de $\omega=\varepsilon k$ es la vorticidad a escala local (regional). No es necesario utilizar funciones de amortiguación en subcapas viscosas porque se han realizado correcciones de bajo número de Reynolds en el coeficiente de viscosidad de Foucault $\mu$ t. Además, el modelo k- $\omega$ se ha adoptado para las capas límites alrededor de las superficies de las paredes [14].

Tomboulides et al. [15] expresan en su investigación que el modelo $k-\omega$ es más utilizado para flujos de régimen turbulento y se encuentra relacionado con uno de los primeros modelos cerrados de turbulencia, $\mathrm{k} 1{ }^{*} 2-\omega$, propuesto originalmente por Kolmogorov (1942). Es un modelo que resuelve dos ecuaciones de transporte para representar propiedades de turbulencia, una para la energía cinética turbulenta (TKE), k, y otra para la tasa de disipación específica (SDR), w. La cantidad $\omega$ está relacionada con la inversa de la escala de tiempo de la turbulencia, mientras que $\mathrm{k}$ representa la energía cinética turbulenta.

\section{F. Modelo SST $k-\omega$}

El modelo SST (Shear Stress Transport), según [16], es un modelo de turbulencia de viscosidad de Foucault de ecuaciones que combina los méritos de ambos $k-\varepsilon$ y el $k-\omega$ que varían formulando fuera de la capa límite mediante funciones de fusión, dependiendo de la escala de longitud de turbulencia. La adopción de un k$\omega$ en los regímenes internos de la capa límite permite aplicar directamente el modelo hasta la pared, mientras que la adopción de capa $k-\varepsilon$ en los regímenes externos elimina la desventaja de la sensibilidad a las propiedades de turbulencia de la corriente libre de entrada. 
En la investigación realizada por Devolver et al. [17], se describe que el modelo SST causa un importante amortiguamiento de las olas para evitar la propagación de las ondas de alta inclinación. Esta amortiguación es provocada por un aumento de la viscosidad turbulenta alrededor del agua y el aire. Este aumento es inducido por la gran producción de energía cinética turbulenta (TKE), k.

La producción de TKE está vinculada al gradiente de velocidad, que es grande alrededor de la interfaz entre el agua y el aire, debido a la velocidad de aire. En consecuencia, existe un desequilibrio natural entre el gradiente de presión y el gradiente de la gran densidad en la superficie del agua libre, a causa de la gran relación de densidad (1000/1). El modelo k- $\omega$ SST está modificado por flotabilidad y se define como:

$$
\begin{gathered}
\frac{\partial \rho \kappa}{\partial \mathrm{t}}+\frac{\partial \rho \mathrm{u}_{\mathrm{i}} \mathrm{k}}{\partial \mathrm{x}_{\mathrm{j}}}-\frac{\partial}{\partial \mathrm{x}_{\mathrm{j}}}\left[\rho\left(\mathrm{v}+\sigma_{\mathrm{k}} \mathrm{V}_{\mathrm{t}}\right) \frac{\partial \mathrm{k}}{\partial \mathrm{x}_{\mathrm{j}}}\right]=\rho \mathrm{P}_{\mathrm{k}}+\mathrm{G}_{\mathrm{b}}-\rho \beta \cdot \omega \mathrm{k} \\
\frac{\partial \rho \kappa}{\partial \mathrm{t}}+\frac{\partial \rho \mathrm{u}_{\mathrm{i}} \mathrm{k}}{\partial \mathrm{x}_{\mathrm{j}}}-\frac{\partial}{\partial \mathrm{x}_{\mathrm{j}}}\left[\rho\left(\mathrm{v}+\sigma_{\omega} \mathrm{V}_{\mathrm{t}}\right) \frac{\partial \omega}{\partial \mathrm{x}_{\mathrm{j}}}\right]=\frac{\gamma}{\mathrm{v}_{\mathrm{t}}} \rho \mathrm{G}-\rho \beta \omega^{2}+2\left(1-\mathrm{F}_{1}\right) \rho \frac{\sigma_{\omega 2}}{\omega} \cdot \frac{\partial \mathrm{k}}{\partial \mathrm{x}_{\mathrm{j}}} \cdot \frac{\partial \omega}{\partial \mathrm{x}_{\mathrm{j}}}
\end{gathered}
$$

Donde: $D_{\omega}$, representa el término de difusión cruzada.

\section{G. Modelo BSL $k-\omega$}

El modelo k- $\omega$ Baseline (BSL), introducido por Tharwat et al. [18], es similar al modelo $k-\omega$ original de Wilcox, sin su fuerte dependencia de los valores de flujo libre arbitrarios. El modelo BSL es idéntico al modelo Wilcox en la región de la capa límite, cambia gradualmente al modelo $k-\varepsilon$ estándar en la región interior de la corriente libre. El modelo BSL es una versión preliminar del modelo Shear Stress Transport (SST). Los resultados del modelo BSL son comparables a los del modelo k- $\omega$ original, sin la dependencia indeseable de flujo libre.

$$
\begin{aligned}
& \frac{\partial}{\partial t}(\rho \kappa)+\frac{\partial}{\partial x_{j}}\left(\rho \kappa \mu_{j}\right)=\frac{\partial}{\partial x_{j}}\left[\left(\Gamma_{\kappa} \frac{\partial \kappa}{\partial x_{j}}\right)\right]+G_{\kappa}-Y_{\kappa}+S_{\kappa} \\
& \frac{\partial}{\partial t}(\rho \omega)+\frac{\partial}{\partial x_{j}}\left(\rho \omega \mu_{j}\right)=\frac{\partial}{\partial x_{j}}\left[\left(\Gamma_{\omega} \frac{\partial \omega}{\partial x_{j}}\right)\right]+G_{\omega}-Y_{\omega}+S_{\omega}
\end{aligned}
$$


Según Mora [19], en estas ecuaciones, $G_{\omega}$ representa la generación de $\omega ; \Gamma_{\kappa}$ y $\Gamma_{\omega}$ es la difusividad efectiva de $\kappa$ y $\omega$, respectivamente; $Y_{\kappa}$ y $Y_{\omega}$ es la disipación de $\kappa$ y $\omega$ debido a la turbulencia.

La efectividad difusiva para $\kappa$ y $\omega$ se obtiene con los siguientes modelos:

$$
\begin{aligned}
& \Gamma_{\kappa}=\mu+\frac{\mu_{t}}{\sigma_{\kappa}} \\
& \Gamma_{\omega}=\mu+\frac{\mu_{t}}{\sigma_{\omega}}
\end{aligned}
$$

\section{Resultados}

Los datos obtenidos de comparación (turbulencia de disipación y turbulencia cinética energética), se realizaron en el software Fluent de ANSYS, teniendo en cuenta las siguientes condiciones: velocidad de entrada del agua de $2.5 \mathrm{~m} / \mathrm{s}$, constante, temperatura en la entrada de $400 \mathrm{~K}$ y temperatura de salida de $303 \mathrm{~K}$. La forma característica de un codo estándar de $90^{\circ}$ lo vuelve más susceptible a turbulencia, en el análisis con el modelo k- $\varepsilon$ estándar, se verificó esta hipótesis en la turbulencia de disipación y energía cinética como se muestra en la Figura 1(a). Por su parte, el doblez posee una turbulencia de disipación muy pequeña con relación al codo, por tal razón no es apreciable en la Figura 1(b). Dado que Fluent CFD aplica un mismo rango de medición para los dos $\left(\min .=0.107126\left[\mathrm{~m}^{2} / \mathrm{s}^{3}\right]\right.$; máx.=113.732 $\left[\mathrm{m}^{2} / \mathrm{s}^{3}\right]$ ), en donde el rango establecido no detecta la turbulencia en el doblez, sin embargo, disminuyendo el rango sí detecta turbulencia en los dos casos. 


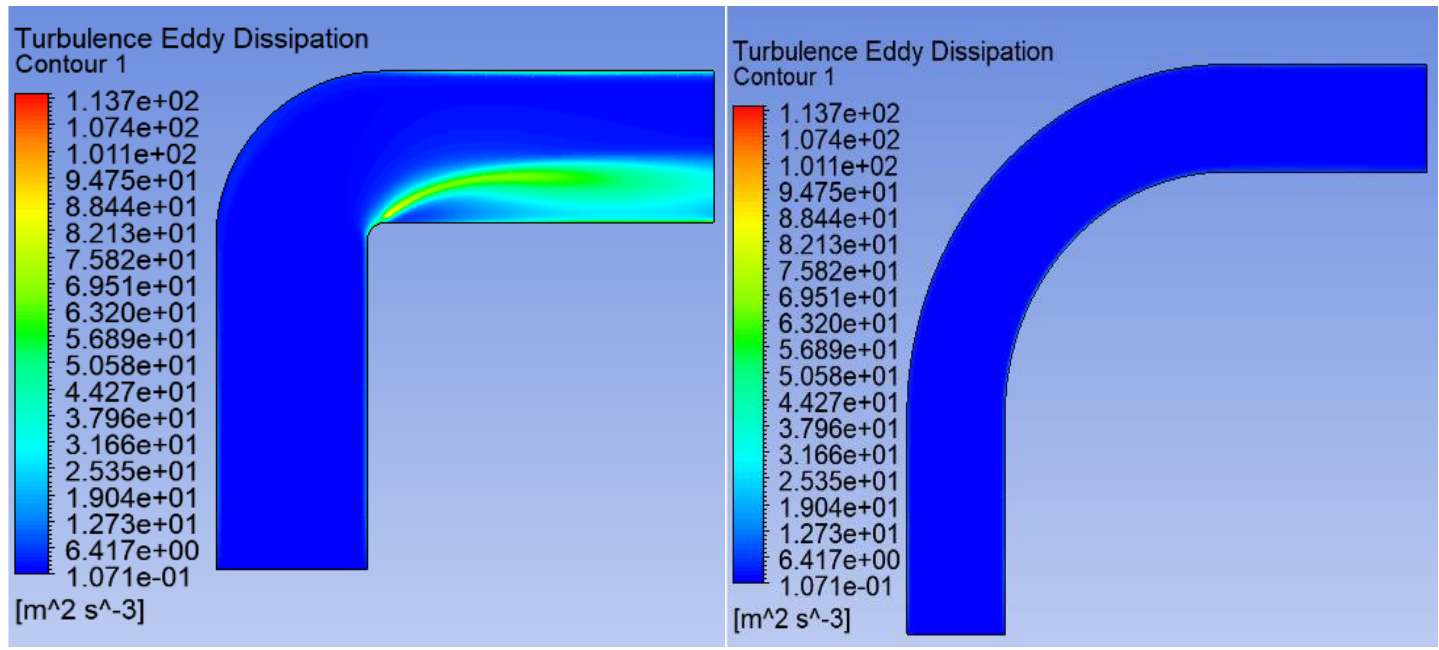

Fig. 1. Turbulencia de disipación k-£ estándar (a) Izquierda (b) Derecha.

En el modelo k- $\varepsilon$ RNG, la turbulencia en el codo es predominante en relación con el doblez, como se observa en la Figura 2(a). Sin embargo, el modelo k- $\varepsilon$ RNG presenta menor turbulencia que el modelo k- $\varepsilon$ estándar, debido a una técnica de doble expansión, mientras que el k- $\varepsilon$ estándar se encuentra regido por variaciones de velocidad, impulso y energía.
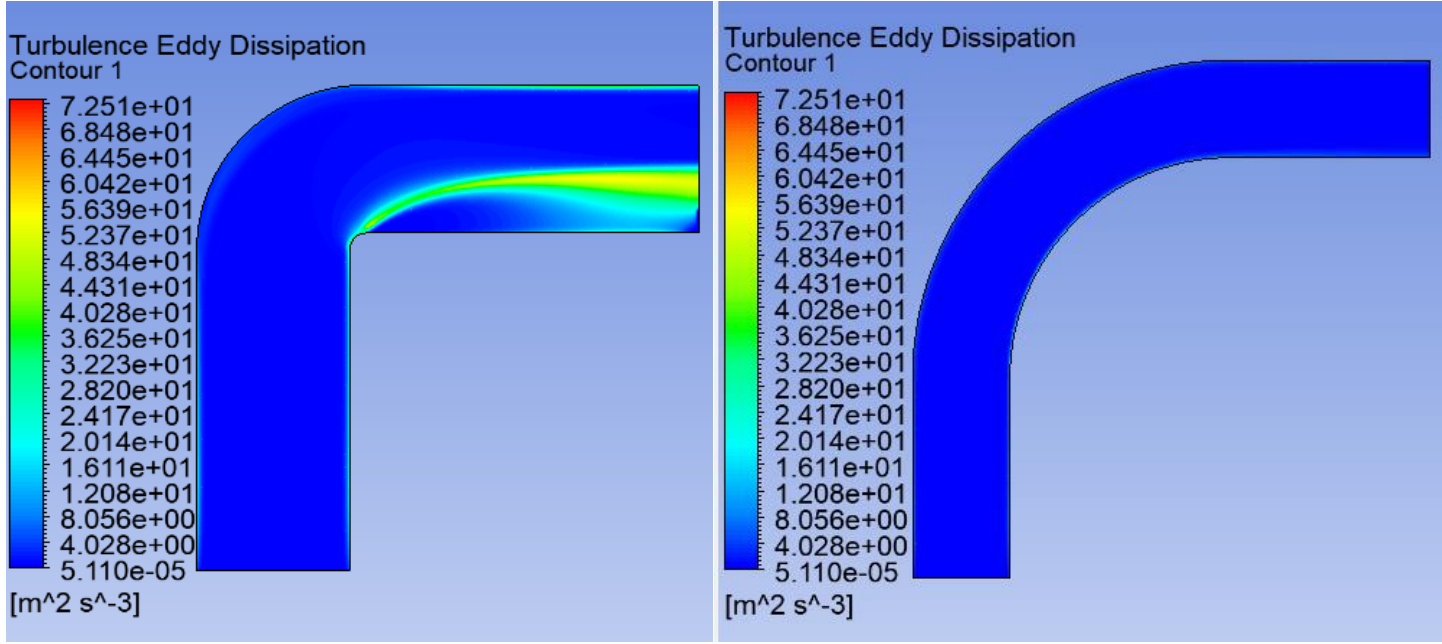

Fig. 2. Turbulencia de disipación k-є RNG (a) Izquierda (b) Derecha.

Tanto la turbulencia de energía de disipación como la turbulencia de energía cinética $k-\varepsilon$ en el codo y doblez poseen una curva con una tendencia creciente con respecto al eje de las abscisas (distancia), como se observa en la Figura 3 (a) y 
Estudio numérico y comparativo del efecto de turbulencia en codos y dobleces para distribución de agua sanitaria

(c), en la cual el método $\mathrm{k}$ - $\varepsilon$ estándar presenta el punto más alto para ambos accesorios.

Los métodos que proponen una turbulencia de energía cinética menor son RNG y realizable para doblez, Figura 3(b), y codo, Figura 3(d), respectivamente.

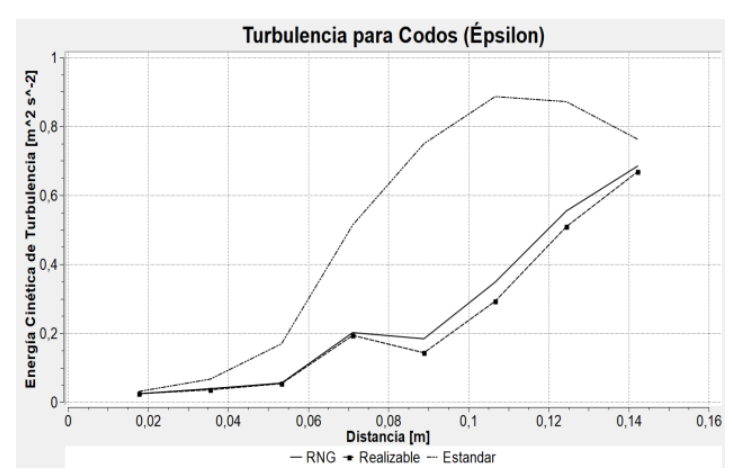

(a)

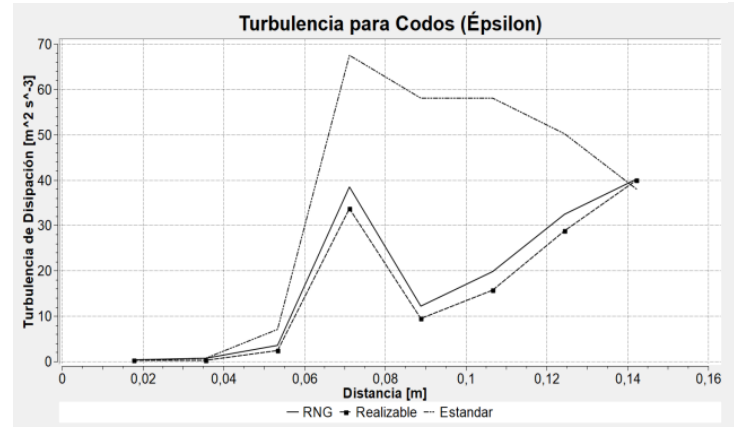

(c)

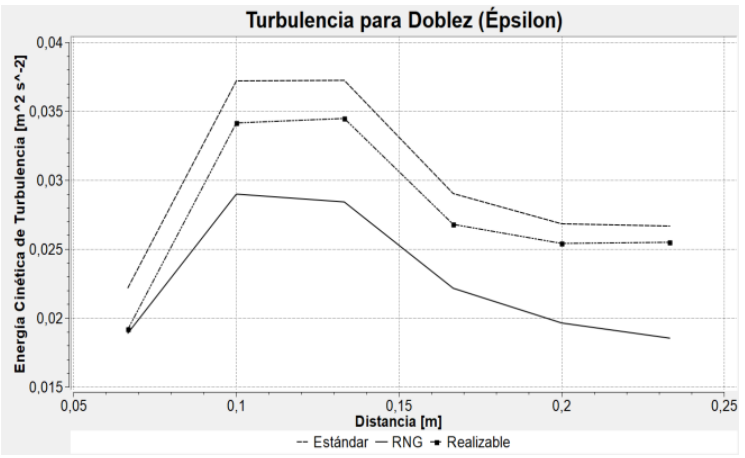

(b)

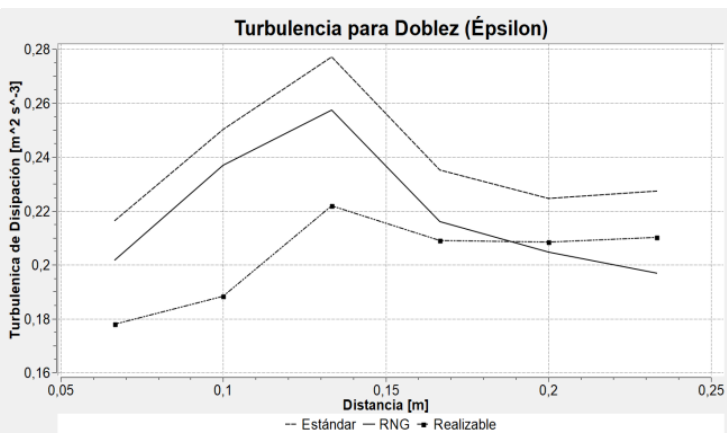

(d)

Fig. 3. Energía cinética de turbulencia y turbulencia de disipación k- $\varepsilon$ estándar, RNG y realizable:

(a), (c) y codo (b), (d) doblez.

A diferencia del modelo k-épsilon, el k-omega no tiene turbulencia de disipación; no obstante, posee turbulencia de frecuencia, siendo mayor que la turbulencia de energía cinética, como se observa en las figuras 4 y 5.

La turbulencia frecuencia en el doblez es baja, solo es apreciable en las paredes de este (Figura 4b). 


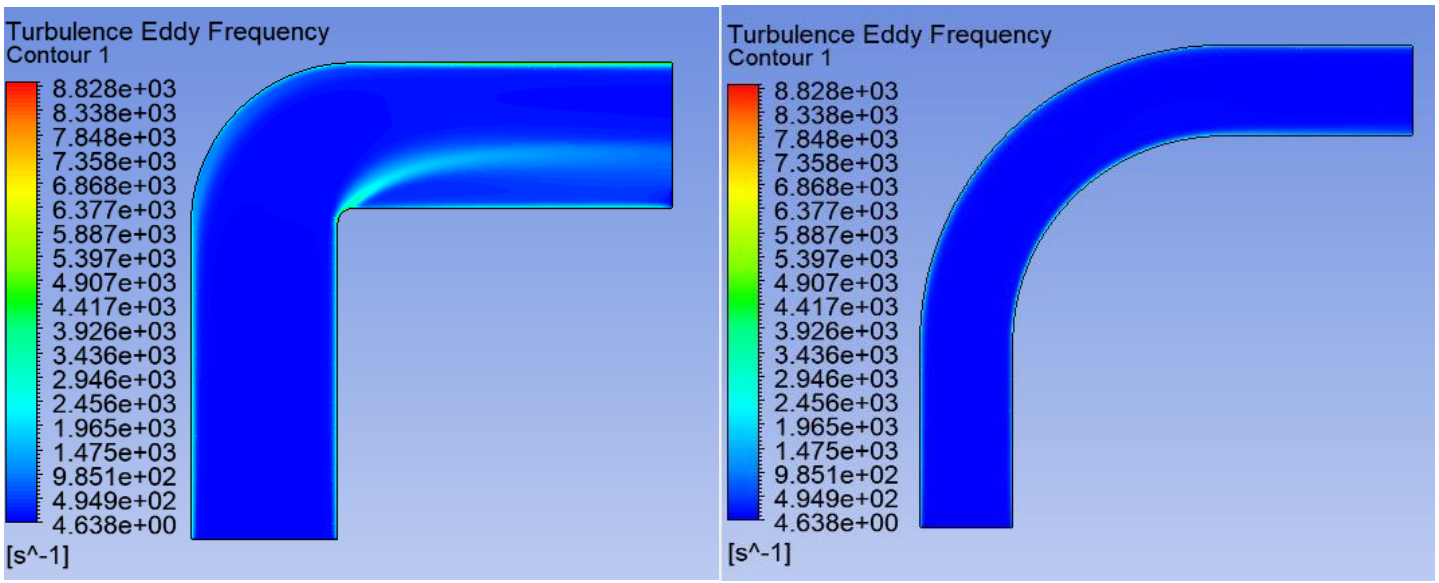

Fig. 4. Turbulencia de frecuencia k- w estándar (a) Izquierda (b) Derecha.

En el caso de la energía cinética en el codo y doblez, se comporta de la misma manera que en el método estándar k-épsilon, puesto que la turbulencia generada por el doblez es muy baja comparada con la del codo (no presenta turbulencia dentro del rango), como se muestra en la Figura 5(b).

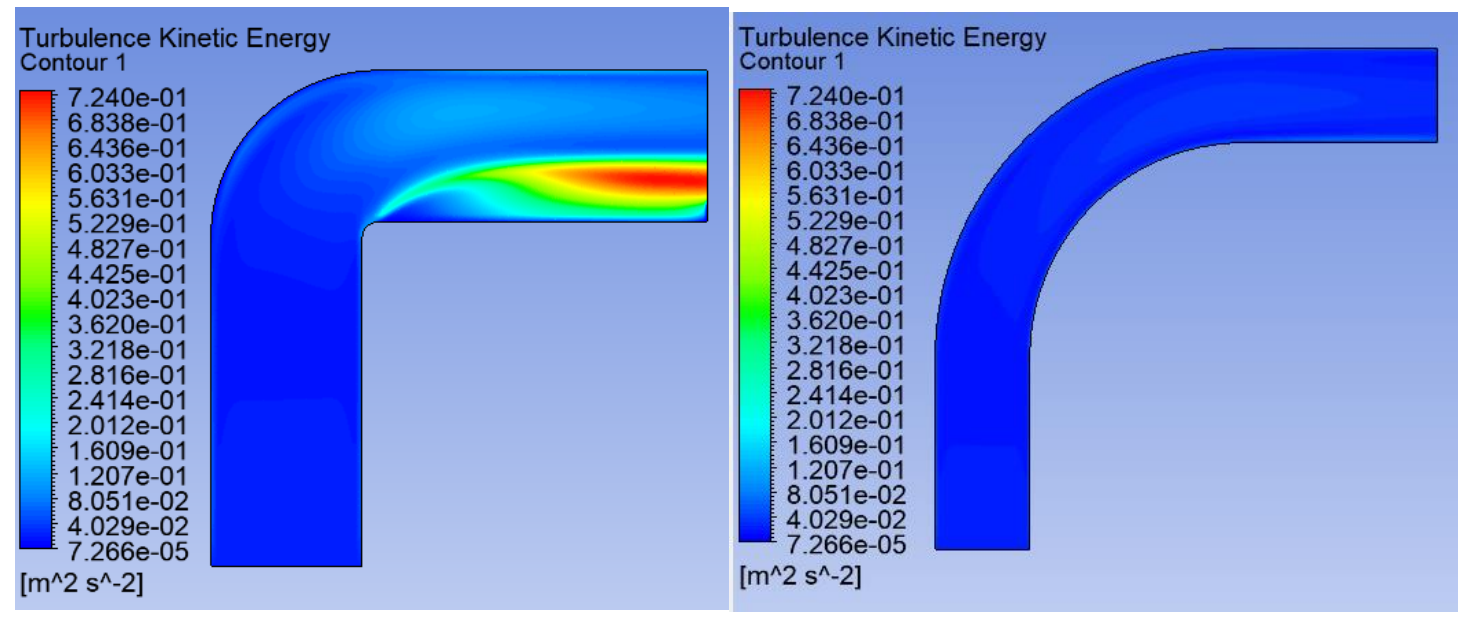

Fig. 5. Turbulencia de energía cinética k- $w$ estándar (a) Izquierda (b) Derecha.

Las curvas de turbulencia de frecuencia tienen como valores pico $1800 \mathrm{~s}^{-1}$ para el codo (Figura 6a) y $104 \mathrm{~s}^{-1}$ para el doblez (Figura 6b), ambos con el método estándar, es decir, la turbulencia de frecuencia k- $\omega$ en el método estándar es 17,31 veces mayor en el codo con respecto al doblez.

Los métodos k-w estándar para el codo (Figura 7a) y SST para el doblez (Figura $7 b)$, representan una menor turbulencia de energía cinética en comparación con Revista Facultad de Ingeniería (Rev. Fac. Ing.) Vol. 28 (53), pp. 101-118. Octubre-Diciembre 2019. 
Estudio numérico y comparativo del efecto de turbulencia en codos y dobleces para distribución de agua sanitaria

los métodos estándar y BSL; es así como se reduce la turbulencia en un $73.68 \%$ y $72.22 \%$ para codo y doblez, respectivamente.

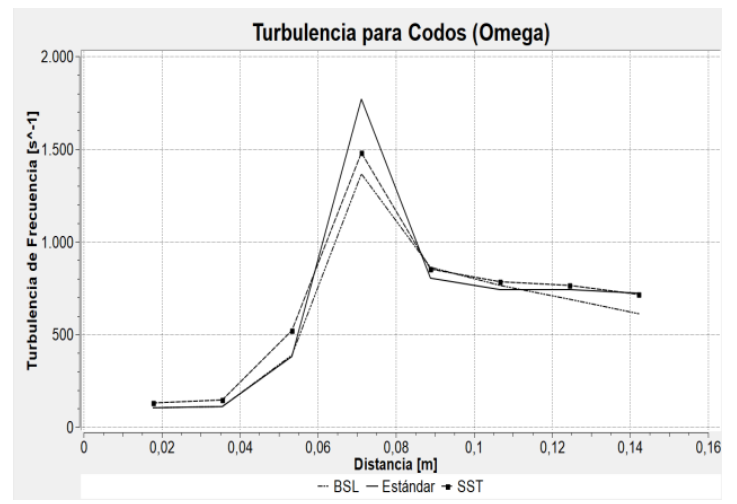

(a)

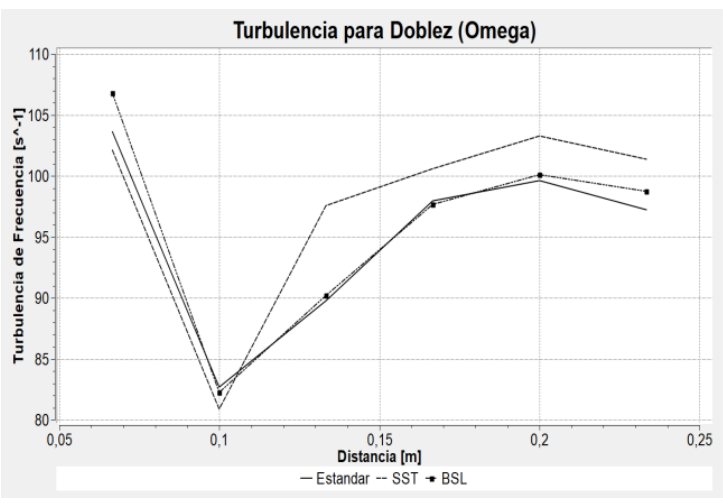

(b)

Fig. 6. Turbulencia de frecuencia k-w estándar, BSL, SST para: (a) codo y (b) doblez.

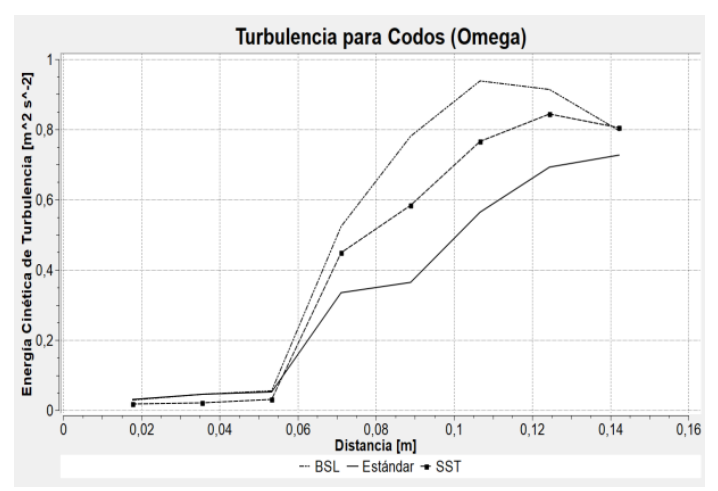

(a)

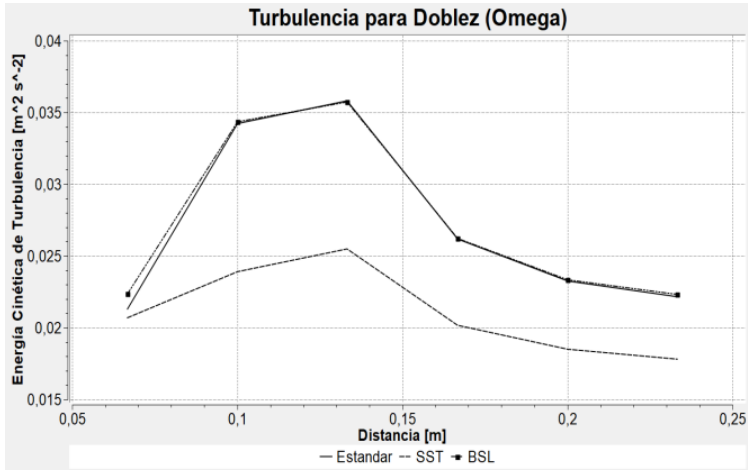

(b)

Fig. 7. Energía cinética de turbulencia k-w estándar, BSL, SST para: (a) codo y (b) doblez.

\section{DISCUSIÓN Y CONCLUSIONES}

Los modelos kappa-omega y kappa-épsilon muestran resultados similares respecto a la turbulencia y a la energía cinética turbulenta, que se presentan en el interior de un codo y doblez. Ambos apuntan a que el codo, debido a su ángulo de $90^{\circ}$ es más propenso a tener cambios en la velocidad, impulso y presión, ya que su geometría no permite que los remolinos creados en el fluido se distribuyan a lo largo de su superficie interna y, por lo tanto, exista turbulencia previa al cambio de dirección. 
En el modelo kappa-épsilon (estándar), la geometría del doblez permite obtener una turbulencia de disipación mínima distribuida a lo largo de las superficies internas, teniendo así un rango de turbulencia entre 1.28 a $2.906\left[\mathrm{~m}^{2} / \mathrm{s}^{3}\right]$, en contraste con el codo con un valor de 24.7 a $73.84\left[\mathrm{~m}^{2} / \mathrm{s}^{3}\right]$, es decir, el codo presenta una turbulencia de disipación 23.54 veces mayor que la del doblez.

El modelo kappa-omega (estándar) es más acertado que el modelo kappa-épsilon, ya que abarca todos los valores en un mismo rango de la turbulencia de frecuencia de 77.5351 a $2306.92\left[\mathrm{~s}^{-1}\right.$ ], donde el codo genera un valor máximo de $1427\left[\mathrm{~s}^{-1}\right.$ ] y el doblez 813.8 [s $^{-1}$ ], por lo tanto, se observa en el codo un $42.97 \%$ más de turbulencia que en el doblez.

En la turbulencia de energía cinética, el modelo kappa-épsilon (RNG) tiene valores máximos de $0.5924\left[\mathrm{~m}^{2} / \mathrm{s}^{2}\right]$ y $0.05535\left[\mathrm{~m}^{2} / \mathrm{s}^{2}\right]$ para el codo y el doblez respectivamente; por el contrario, el modelo kappa-omega (BSL) presenta cantidades de $1.136\left[\mathrm{~m}^{2} / \mathrm{s}^{2}\right]$ y $0.05565\left[\mathrm{~m}^{2} / \mathrm{s}^{2}\right]$. Con base en los resultados se observa que en BSL genera $47 \%$ más turbulencia de energía cinética en el codo y $0.53 \%$ en el doblez.

\section{CONTRIBUCIÓN DE LOS AUTORES}

La idea principal y el desarrollo estuvieron a cargo de Luis Fernando Toapanta; el análisis posterior lo llevaron a cabo Cholango y Zapata; la verificación de resultados de la simulación fue hecha por Quitiaquez, Nieto-Londoño y Zapata.

\section{AGRADECIMIENTOS}

Los autores agradecen de manera especial al Grupo de Investigación de Energías Renovables e Implementación Mecánica de Pymes de la Universidad Politécnica Salesiana, de Quito, Ecuador, y a la Universidad Pontificia Bolivariana, de Medellín, Colombia, por sus grandes aportes a esta investigación.

\section{REFERENCIAS}

[1] J. Jiménez, "Turbulence structure and vortex dynamics," Eur. J. Mech. - B/Fluids., vol. 20 (5), pp. 746747, Sep. 2001. https://doi.org/10.1016/s0997-7546(01)01149-9.

[2] L. Davidson. Fluid mechanics, turbulent flow and turbulence modeling, CFD Course, pp. 1-270, 2012. 
Estudio numérico y comparativo del efecto de turbulencia en codos y dobleces para distribución de agua sanitaria

[Online]. Available at: https://es.scribd.com/document/321777933/Fluid-mechanics-turbulent-flow-andturbulence-Lars-Davidson-pdf.

[3] P. Dutta, S. K. Saha, N. Nandi, and N. Pal, "Numerical study on flow separation in $90^{\circ}$ pipe bend under high Reynolds number by k- $\varepsilon$ modelling," Engineering Science and Technology an International Journal, vol. 19 (2), pp. 904-910, Jun. 2016. https://doi.org/10.1016/j.jestch.2015.12.005.

[4] Y. Ikarashi, T. Uno, T. Yamagata, and N. Fujisawa, "Influence of elbow curvature on flow and turbulence structure through a 90 elbow," Nuclear Engineering and Design, vol. 339, pp. 181-193, Dec. 2018. https://doi.org/10.1016/i.nucengdes.2018.09.011.

[5] D. Wang, D. Ewing, and C. Y. Ching, "Time evolution of surface roughness in pipes due to mass transfer under different Reynolds numbers," International Journal of Heat and Mass Transfer, vol. 103 pp. 661671, Dec. 2016. https://doi.org/10.1016/i.ijheatmasstransfer.2016.08.004.

[6] M. Zamani, S. Seddighi, and H. R. Nazif, "Erosion of natural gas elbows due to rotating particles in turbulent gas-solid flow," Journal of Natural Gas Science and Engineering, vol. 40, pp. 91-113, Apr. 2017. https://doi.org/10.1016/i.jngse.2017.01.034.

[7] N. Lin, H. Lan, Y. Xu, S. Dong, and G. Barber, "Effect of the gas-solid two-phase flow velocity on elbow erosion," Journal of Natural Gas Science and Engineering, vol. 26, pp. 581-586, Sep. 2015. https://doi.org/10.1016/i.jngse.2015.06.054.

[8] A. Zacarias, A. González, J. Granados, and A. Mota. Mecánica de fluidos, teoría con aplicación y moldeado. México: Patria, 2017.

[9] C. Rumsey, Modelos de turblencia: RANS y LES. s.I.: s.n., s.f.

[10] C. A. Bayona, L. P. Londoño, and E. A. Nieto, "Identificación del modelo de turbulencia más adecuado, utilizando software de dinámica de fluidos computacional, para el diseño del vehículo urbano de la competencia Shell Eco Marathon," Grade Thesis, Universidad de San Buenaventura, Bogotá D.C., Colombia, 2015.

[11] L. F. Toapanta-Ramos, A. G. Bohórquez, L. E. Caiza, and S. Quitiaquez, " Numerical analysis of the speed profiles of a water flow through a gradual reduction pipe," Enfoque UTE, vol. 9 (3), pp. 80-92, Sep. 2018. https://doi.org/10.29019/enfoqueute.v9n3.290.

[12] P. Mishra, and K.R. Aharwal, "A review on selection of turbulence model for CFD analysis of air flow within a cold storage," IOP Conf. Ser. Mater. Sci. Eng., vol. 402, pp. 012145-012153, Sep. 2018. https://doi.org/10.1088/1757-899X/402/1/012145.

[13] J. Shih, L. Tsan-Hsing, W. William, S. Aamir, Y. Zhigang, and J. Zhu, "A new eddy viscosity model for high Reynolds number turbulent flows," Computer Fluids, vol. 24 (3), pp. 227-238, 1995. https://doi.org/10.1016/0045-7930(94)00032-t.

[14] K. Ito, K. Inthavong, T. Kurabuchi, T. Ueda, T. Endo, T. Omori, H. Ono, S. Kato, K. Sakai, Y. Suwa, H. Matsumoto, H. Yoshino, W. Zhang, and J. Tu, "CFD Benchmark Tests for Indoor Environmental Problems: Part 1 Isothermal/Non-Isothermal Flow in 2D and 3D Room Model," Int. J. Archit. Eng. Technol., vol. 2 (1), pp. 01-22, Apr. 2015. https://doi.org/10.15377/2409-9821.2015.02.01.1.

[15] A. Tomboulides, S. M. Aithal, P. F. Fischer, E. Merzari, A. V. Obabko, and D. R. Shaver, "A novel numerical treatment of the near-wall regions in the $k-\omega$ class of RANS models," International Journal of Heat and Fluid Flow, vol. 72, pp. 186-199, Aug. 2018. https://doi.org/10.1016/j.ijheatfluidflow.2018.05.017.

[16] K. An, and J. C. H. Fung, "An improved SST k- $\omega$ model for pollutant dispersion simulations within an Revista Facultad de Ingeniería (Rev. Fac. Ing.) Vol. 28 (53), pp. 101-118. Octubre-Diciembre 2019. Tunja-Boyacá, Colombia. L-ISSN: 0121-1129, e-ISSN: 2357-5328, DOI: https://doi.org/10.19053/01211129.v28.n53.2019.10156 
Luis Fernando Toapanta-Ramos, Jorge Alejandro Zapata-Cautillo, Andrea Isabel CholangoGavilanes, William Quitiaquez, César Nieto-Londoño, Zulamita Zapata-Benabithe

isothermal boundary layer," Journal of Wind Engineering and Industrial Aerodynamics, vol. 179, pp. 369384, Aug. 2018. https://doi.org/10.1016/j.jweia.2018.06.010.

[17] B. Devolder, P. Troch, and P. Rauwoens, "Performance of a buoyancy-modified k- $\omega$ and k- $\omega$ SST turbulence model for simulating wave breaking under regular waves using OpenFOAM®," Coastal Engineering, vol. 138, pp. 49-65, Aug. 2018. https://doi.org/10.1016/i.coastaleng.2018.04.011.

[18] A. Tharwat, R. El-Samanoundy, and M. El-Baz, "Considerations of Stress Limiter for the SST Turbulence Model in Dual Throat Nozzle Predictions," in Ninth International Conference on Computational Fluid Dynamics (ICCFD9), Istanbul, Turkey, 2016, pp.1-15.

[19] J. A. Mora, and G. R. Santos, "Modelación hidrodinámica Bi y Tridimensional de dos canales con disipador de energía del laboratorio de la Escuela Colombiana de Ingeniería utilizando Ansys Fluent," Master Thesis, Escuela Colombiana de Ingeniería, Bogotá D. C., Colombia, 2018.

Revista Facultad de Ingeniería (Rev. Fac. Ing.) Vol. 28 (53), pp. 101-118. Octubre-Diciembre 2019.

Tunja-Boyacá, Colombia. L-ISSN: 0121-1129, e-ISSN: 2357-5328, DOI:

https://doi.org/10.19053/01211129.v28.n53.2019.10156 